\title{
Miranda
}

Revue pluridisciplinaire du monde anglophone /

Multidisciplinary peer-reviewed journal on the English-

speaking world

$10 \mid 2014$

Images on the Move: Circulations and Transfers in film

\section{De la pellicule au pixel. À propos des remédiations numériques de films expérimentaux sur Internet (et YouTube en particulier)}

\section{André Habib}

\section{OpenEdition}

\section{Journals}

Édition électronique

URL : http://journals.openedition.org/miranda/6312

DOI : 10.4000/miranda.6312

ISSN : 2108-6559

\section{Éditeur}

Université Toulouse - Jean Jaurès

\section{Référence électronique}

André Habib, « De la pellicule au pixel. À propos des remédiations numériques de films expérimentaux sur Internet (et YouTube en particulier) », Miranda [En ligne], 10 | 2014, mis en ligne le 23 février 2015, consulté le 16 février 2021. URL : http://journals.openedition.org/miranda/6312 ; DOI : https://doi.org/ $10.4000 /$ miranda. 6312

Ce document a été généré automatiquement le 16 février 2021.

\section{cc)}

Miranda is licensed under a Creative Commons Attribution-NonCommercial-NoDerivatives 4.0

International License. 


\title{
De la pellicule au pixel. À propos des remédiations numériques de films expérimentaux sur Internet (et YouTube en particulier)
}

\author{
André Habib
}

Si d'aventure il venait à l'idée d'un visiteur de YouTube d'inscrire les mots «Mothlight » dans le moteur de recherche du site, il tomberait, parmi d'autres choses (des clips du groupe pop Mothlight, une vidéo d'un entomologiste), sur plusieurs « versions » d'un film célèbre de l'histoire du cinéma expérimental, réalisé par Stan Brakhage en 1963, Mothlight. Il est sans doute difficile de décrire, particulièrement pour ceux qui ont fait l'expérience du film en projection $16 \mathrm{~mm}$, le degré de défiguration qu'il subit en passant dans le rouleau compresseur du numérique : pixélisé à outrance, heurté de balayements noirs, aux couleurs délavées, uniformisées dans un même grisvert terreux, fruit d'un transfert numérique, mal exposé - en somme, des plus douteux. À cela s'ajoute les distractions usuelles qui naissent dans le voisinage ou directement sur l'image du film (publicités, icônes, barre de défilement, etc.), toutes bien étrangères à la vision du cinéaste américain. Pour mémoire, on se rappellera que Mothlight a été bricolé en appliquant directement, entre deux bandes adhésives, sur de la pellicule $16 \mathrm{~mm}$ transparente, des ailes de phalènes, des feuilles d'arbres découpées, des fines tiges, des lames de gazon, de telle sorte que par l'action du défilement de la pellicule et de la lumière du projecteur, ces natures mortes se trouvent en quelque sorte réanimées; leur mort, rédimées par l'art du cinéma. Avec Le retour à la raison (1923) de Man Ray comme illustre ancêtre (du moins pour une bonne part des images), et bien d'autres depuis, réalisés par Brakhage (on pense à The Garden of Earthly Delights, 1981) ou qui lui rendent directement hommage (comme le film Papillon, 2006, d'Olivier Fouchard), il s'agit de films réalisés «sans caméra » (bien qu'avec l'aide d'une tireuse optique). En se servant de la pellicule comme surface d'inscription, comme une toile en quelque sorte, et non comme une bande découpée en séquence d'unités isolées, Mothlight défie la logique photogrammatique du cinéma, produisant ainsi un 
papillonnement stroboscopique qui fait toute la puissance esthétique et conceptuelle du film, jouant entre figuration on ne peut plus concrète (ce sont les choses ellesmêmes qui apparaissent sous nos yeux) et abstraction (ces choses passent tellement vite qu'on n'a pas le temps de les voir distinctement). Tout ceci ne peut qu'être violenté par un transfert numérique, et particulièrement dans une version hautement compressée, comme celle que l'on trouve sur YouTube ${ }^{1}$.

Il s'agit très certainement de l'un des 25 films expérimentaux de la seconde moitié du vingtième siècle les plus connus et marquants, aux côtés de Wavelength (1967) de Michael Snow, ou Arnulf Rainer (1959) de Kubelka (titres que j'ai nommés à loisir puisque ce sera sur eux que je m'appuierai tout au long de cet article, même si Empire (1963) ou Blowjob (1964) de Warhol, ou encore The Flicker (1966) de Tony Conrad sont aussi de bons exemples). Mothlight est en général un bon film pour introduire des étudiants de cinéma à une certaine puissance $-\mathrm{y}$ compris de défamiliarisation $-\mathrm{du}$ cinéma expérimental, tout en permettant de les exposer aux spécificités matérielles du cinéma. Quand je dispense un cours sur le cinéma expérimental, il m'arrive, après avoir projeté la bobine, de la faire dérouler en classe afin que les étudiants puissent concrètement voir de quoi le film qu'ils viennent de voir est fait, un peu à la manière du cinéaste autrichien Peter Kubelka qui déroule régulièrement les bobines de ses films entre les mains du public. Le statut de Mothlight, et les très nombreuses remédiations ${ }^{2}$ auxquelles le film a donné lieu sur des sites comme YouTube ou en DVD, en fait également un bon objet à présenter à des jeunes personnes dont la familiarité la plus immédiate avec le cinéma passe par la circulation du cinéma sur support numérique (je diffuse les différentes versions numériques avant de leur présenter le film en $16 \mathrm{~mm}$ ). Ma version, mes versions YouTube de Mothlight (car il en existe de nombreuses, et à force de m'y intéresser, j'ai fini par me les approprier) opèrent comme des repoussoirs, des sortes de gri-gri ou de talisman, voués à convaincre une fois pour toutes les étudiants des méfaits irréparables de la compression numérique et de bien distinguer ces non-versions des « vraies » et seules expériences « légitimes » de ces films, dans une belle copie en $16 \mathrm{~mm}$, dans une salle obscure, avec un bon projecteur. C'est à ce prix que ce film, et que bon nombre de films expérimentaux acquièrent, je m'époumone à le leur expliquer, une lisibilité, une intelligibilité. De la même manière, personne n'accepterait de voir exposée une reproduction d'un Picasso dans un musée, et ce, malgré les vertus pédagogiques - versant musée imaginaire - que les reproductions peuvent générer, it is simply not the " real » thing.

Cela étant dit, et malgré tout l'acharnement que je mets à défendre le visionnement du cinéma, et du cinéma expérimental en particulier, sur son support d'origine (j'ai l'immense privilège d'enseigner dans un département qui m'accorde un budget de location de film en $16 \mathrm{~mm}$ ), je ne cesse d'être fasciné depuis des années, et ce texte en est le symptôme névrotique, par la présence paradoxale de ces remédiations sur le Web, et en particulier sur YouTube. Elles me semblent révélatrices et symptomatiques des modalités de la circulation des images contemporaines, des effets de communauté paradoxaux qu'ils induisent, de la culture de réappropriation, de parodie, de détournement dans laquelle elle s'inscrit. Au-delà de leur caractère repoussant et de leur valeur repoussoir, j'ai fini, en m'y attardant, par leur trouver une dimension productive sur plusieurs plans, et qui ne sont pas étrangers aux enjeux de cette étude. 


\section{Expérience de la pauvreté : cultures de la basse résolution}

4 Il existe depuis quelques années des versions qu'on pourrait dire admissibles, en DVD et en Blu-Ray, d'œuvres clés du cinéma expérimental, des films qui auparavant étaient voués à une invisibilité quasi-complète autrement que par les circuits légitimes de la distribution en $16 \mathrm{~mm}$. Ces DVD ont permis de rendre visibles, depuis une dizaine d'années tout au plus, des pans entiers d'œuvres dont l'accès se limitait bien souvent à des descriptions parfois peu fiables d'historiens et de critiques : les films de Brakhage, Frampton, Sherwin, Tscherkassky, Kren, Mekas, sont aujourd'hui distribués par des compagnies respectées comme Criterion, Index, Lux, Re:Voir, ou Potemkine. Ces éditeurs se sont lancés dans la production d'éditions tout à fait dignes, dotées d'un appareil critique érudit, documenté, notamment sur les opérations de transferts, et souvent accompagnées d'avertissement rappelant l'effet néfaste que ces copies pourraient avoir sur l'appréciation des films. En effet, comme le souligne l'avertissement que le distributeur parisien Re:VOIR faisait circuler il y a quelques années, la compression DVD, en Mpeg2, ne permet pas le report intégral des 24 (ou 18) images / secondes contenues sur une pellicule, outre le fait que l'expérience propre de ces films qui consiste bien souvent en une exploration des propriétés matérielles - sur un versant ou l'autre - de la pellicule ou du dispositif de projection, est totalement escamotée, et encore plus, bien entendu, en passant dans le goulot d'étranglement du Web.

5 Parmi les œuvres apparaissant sur YouTube et qui se révèlent éloquentes sur ce point, on pourrait citer le classique Arnulf Rainer, monument minimaliste de Peter Kubelka réalisé en 1960, ancêtre des flicker films de Conrad ou Sharits, composé de photogrammes blanc et noir alternés, ainsi que de silence et de bruit «blanc». Cette œuvre qui incarne la pureté la plus extrême du cinéma, réduite à ses éléments fondamentaux, n'existe évidemment pas de façon officielle en DVD (Kubelka est un puriste sur ce point). Il reste évidemment peu de choses de l'expérience « réelle », pour ceux qui ont eu la chance de la vivre en $16 \mathrm{~mm}$ ou en $35 \mathrm{~mm}$, de ce film sur YouTube, en particulier dans une version apparue sur le Web le 31 décembre 2007, mise en ligne par un certain Randy Meeks, et qui a été visionnée plus de 28000 fois ${ }^{3}$. À un très douteux téléciné ou simple refilmage, qui semble être passé à cette étape de sa vie par une incarnation en VHS avant d'être numérisé pour le Web, d'une déjà vilaine copie $16 \mathrm{~mm}$ (on constate de nombreuses rayures) projetée par un mauvais projecteur tungsten (la lampe projetant une lumière jaunâtre semble très irrégulière, l'image est très sombre dans les coins), s'ajoute les défauts inhérents à la pixellisation découlant de la compression numérique. C'est évidemment une catastrophe complète, totalement étrangère au type d'assaut de lumière et de son auquel donne lieu ce film. Cet ersatz, pâle succédané du film d'où il tire son origine, a donné lieu à de nombreux commentaires sur YouTube ${ }^{4}$. On y trouve des échanges très drôles et animés, de fans et de détracteurs, un certain nombres de ces échanges portant sur le statut de l'objet et l'écart entre le film et son ombre pixélisée, comme cette remarque, en réaction au commentaire d'une personne décrivant son expérience du film, vu en $35 \mathrm{~mm}$, au MOMA : "What you saw was the film. This is not the film... it's something else... A preview transfer. Still - glad it's here, somewhat. (safetydpt) » L'intérêt que je porte à ce phénomène se situe précisément entre ce «something else » et ce " somewhat ». 
6 Arnulf Rainer est une partition abstraite, quasi-idéale, voire absolutiste, explorant les matières pures du cinéma. Or, ces versions dégradées, appauvries, déclassées de ces films mettent en valeur la " réalité empirique », souvent moins idéale, de la circulation du cinéma en général et de ce cinéma en particulier (et, disons-le, la façon souvent moins glorieuse dont nous les avons nous-mêmes découverts). En tombant sur une version un peu meilleure du même film on constate sans peine, dans l'écart des versions, la singularité de chacune des copies du film, mais également de ces transferts plus ou moins officiels, ramenant l'absolutisme du film à sa réalité matérielle, forcément impure. On peut aussi dire que la compression inhérente au Web tend à faire apparaître le médium numérique dans toute l'évidence de son unité de base, le pixel, un peu, par analogie, à la manière dont Arnulf Rainer se construisait sur les unités de base du cinéma, le photogramme et le son.

\section{La circulation dans tous ses états}

7 Dans un article en hommage aux images pauvres, «In Defense of Poor Images », Hito Seyerl écrit :

8 That rare prints of militant, experimental, and classical works of cinema as well as video art reappear as poor images is significant on another level. Their situation reveals much more than the content or appearance of the images themselves: it also reveals the conditions of their marginalization, the constellation of social forces leading to their online circulation as poor images. Poor images are poor because they are not assigned any value within the class society of images - their status as illicit or degraded grants them exemption from its criteria. Their lack of resolution attests to their appropriation and displacement. [...] The poor image is no longer about the real thing - the originary original. Instead, it is about its own real conditions of existence: about swarm circulation, digital dispersion, fractured and flexible temporalities. It is about defiance and appropriation [...]. (Seyerl 2010)

Ces " pauvres images » sont en effet à l'image de leur statut au sein de l'écologie plus large de la jungle des images (malgré leur immense capital symbolique auprès d'un certain milieu) : déclassées et marginalisées, luttant à la manière d'un petit corps étranger dans un milieu dans lequel il se confond en perdant son identité. L'un des intérêts d'analyser leur apparition sur des sites comme YouTube repose sur le fait que ces images pauvres, plutôt que d'exister en soi en tant qu'image, sont des déictiques, pointant ou rendant visible, dans les interstices et les parasites qu'ils charrient, l'histoire de leur circulation, bien souvent illégitime, voire illégale ; les lieux, les modes et les supports d'enregistrement par lesquels ils ont transité ; et le désir d'un partage communautaire. Je ne suis pas le premier à en faire la remarque, mais il est remarquable de constater que, même pour le cinéma d'avant-garde, le contenu de YouTube a été et demeure majoritairement du contenu télévisuel stocké, parfois bien avant l'arrivée d'Internet, sur des bandes VHS, NTSC ou PAL, usées par les multiples transferts et le passage du temps qui tend à émousser et hérisser d'un bruit électronique les contours de l'image. En s'attardant sur des détails dans la périphérie de l'image, on constate par exemple qu'une des versions disponibles sur YouTube de Mothlight provient d'un repiquage de la télé allemande (on voit d'ailleurs apparaître, au début, un sous-titre, Mottenlicht). Il existe également une version - sans doute la meilleure, et en tout cas la plus « connue » des internautes, apparue le 21 janvier 2012, 
mise en ligne par un usager du nom de «trainsdistance » et vue 35000 fois - du chef d'œuvre du cinéma structurel, Wavelength de Michael Snow. Il faut parfois, avant d'arriver au film (et de plus en plus), attendre le passage d'une publicité, comme pour nous rappeler les forces néocapitalistes qui sont au fondement d'un site comme YouTube, et qui sont une sorte de contre-champ absolu de la radicalité esthétique (et économique) du film de Snow. Après le prélude publicitaire, les 45 minutes de Wavelength sont là. Cette version sur YouTube "archive " pour ainsi dire sa source, grâce au logo, apparaissant en haut à droite : il s'agit en fait d'une version numérique repiquée de la Rai Tre, sûrement du programme de minuit, bien connu des cinéphiles italiens, Fuori orario, programme de Enrico Ghezzi, qui présente depuis de nombreuses années des films hors-norme de toutes sortes. À ce propos, on retrouve également sur la version YouTube de La Région centrale (Snow, 1971) le même logo. Il en va de même de plusieurs autres films expérimentaux que l'on trouve sur YouTube, attestant, par la bande du site, de l'effet déterminant que la chaine a eu dans la circulation du cinéma expérimental auprès des cinéphiles italiens férus d'avant-garde, et des modes clandestins de visionnement de ces œuvres copiées et recopiées.

10 À propos de Wavelength, à côté de la version «intégrale » de 45 minutes, on trouve également un extrait de 9 minutes 46 (les dernières minutes du film), mis en ligne en 2006, visionné 84723 fois $^{5}$, dont la basse résolution numérique, les parasites et les interférences de la vidéo (l'image est constamment traversée de bigarrures horizontales qui tordent l'image et le son, les couleurs sont hérissées) ajoutent leur supplément d'invention et de brouillage aux opérations plastiques de Snow (l'extrait, reprenant l'idée de Seyerl, concerne moins le film de Snow que l'exposition de sa circulation clandestine). Le téléchargement par un internaute d'une image aussi mauvaise, a clairement un caractère symptomatique: il témoigne d'un orgueilleux désir de partage qui a préséance sur ce qui est partagé, comme si on pouvait se contenter de l'ombre de la chose pour provoquer une série d'échanges qui, pour la plupart, renvoient à une autre expérience du film que celle que l'on voit ici. Il n'existe pas, et avec raison je dirais, de copies DVD ou BluRay de Wavelength (l'auteur, comme Kubelka et d'autres, s'y refusent de façon absolue). Aussi, ce film, dont le statut au sein de l'histoire du cinéma expérimental est absolument mythique, s'est propagé grâce à la multiplication de ces mauvais télécinés, sur des cassettes vidéo dégradées, dans des fichiers numériques compressés, etc. Et il est possible de constater que ces versions se trouvent aujourd'hui investies, passage du temps oblige et à l'ère du HD lustré et très net, d'une "mélancolie vidéophilique», selon l'expression de Lucas Hildebrand (Hildebrand 2004 77).

11 Nombres d'œuvres, plus ou moins contemporaines du phénomène YouTube (ou Ubuweb, Karagarga, et autres sites spécialisés en cinéma d'avant-garde), sur lesquelles il serait difficile de s'étendre dans le cadre de cet article, se nourrissent d'ailleurs de cette culture mélancolique de l'esthétique du bootleg, faisant du brouillage visuel et sonore le lieu d'une nouvelle déclinaison de l'aura : apparition non plus « unique » mais « reproduite à l'infini » d'un « lointain ». C'est le cas notamment de Factory Archives de Slater Bradley, une bande vidéo réalisée en 2001-2002 (faisant partie de sa Doppelganger Trilogy), superbe matérialisation esthétique de la culture du bootleg video et des communautés de fans. Dans Factory Archives Bradley traite du culte entourant les très rares documents filmés du groupe Joy Division. En demandant à un ami d'interpréter les mouvements caractéristiques des girations du chanteur Ian Curtis, en ralentissant l'image, en travaillant le son, et en recopiant l'image au point de la défiguration, il 
retrouve le grain opaque, la palette décolorée, monochrome, le son distant, fantomatique, de ces bandes vidéos clandestines que les fans du groupe ont notoirement fait circuler. C'est cette nouvelle définition de l'aura, à l'âge de la reproductibilité magnétique et numérique des images, qu'explore ce film et bien d'autres ${ }^{6}$. On pourrait ajouter que la version sur YouTube de Factory Archives, une œuvre très rare et destinée à un visionnement dans les galeries d'art, est particulièrement mauvaise ${ }^{7}$, ce qui rajoute une couche méta-réflexive de plus, conforme, au fond, à l'esprit de l'œuvre (tout en trahissant sa lettre). Là encore, on prend conscience du médium par le bruit qu'il produit, l'interférence qu'il génère.

Ces copies, et les traces institutionnelles et technologiques qui se sédimentent en elles, permettent de souligner une autre cinéphilie, vers laquelle le simulacre de Bradley pointe : une cinéphilie collectionneuse, fétichisant moins les instants privilégiés dans les œuvres que l'objet matériel (ou dématérialisé), la possession de la chose, et dont la pauvreté visuelle participe de la rareté, et est proportionnelle à son degré de clandestinité. Elle garde, sur sa trame usée, la trace des multiples visionnements, de nombreux successeurs qui à chaque génération, contribuent à un appauvrissement de l'image, à un dépérissement du signal, au point de produire, à toute fin pratique, une nouvelle œuvre, qui a souvent peu à voir avec l'original. Si on reprend l'exemple de l'extrait de 9 minutes de Wavelength, la personne qui l'a placé sur YouTube ne cherchait sans doute pas à rendre un hommage respectueux au film: c'était une manière d'exprimer la rareté de sa possession, de dire " j'ai une copie de Wavelength, pas très bonne, mais je l'ai quand même, voici, je te la montre.» On peut d'ailleurs dire qu'au «j'ai vu» de la cinéphilie d'autrefois, s'est substituée un «je l'ai» ou encore «j'ai vécu » même si ce qui est partagé n'a rien à voir avec l'expérience réelle.

\section{Effets de communauté}

Reprenant l'idée soulignée par Lucas Hildebrand à propos de la circulation du film controversé de Todd Haynes, Superstar (Hildebrand 2004 80-84), on pourrait dire que la circulation des films expérimentaux permet de témoigner du réseau communautaire que ces films, marginalisés ou difficiles d'accès, ont produits, niches ou sectes qui transbahutent, faute de mieux, et avec la passion du partage et l'orgueil du collectionneur, ces terribles copies (on pourrait dire la même chose d'une certaine frange du cinéma d'exploitation ou transgressif). Internet est très certainement venu exacerber cet état de fait en le déplaçant dans l'espace public et en permettant à toutes ces versions illégitimes et souvent franchement dégueulasses de trouver une visibilité publique inattendue, bien que parfois le collectionneur aura tendance à partager son butin dans des «clubs privés » au sein de la toile, comme des sites peer to peer (P2P), hautement surveillés par une communauté de cinéphiles assidus, ultra-spécialisés, comme le site Karagarga (www.karagarga.net), véritable secte réservée aux initiés seulement, auquel il est très difficile d'accéder.

14 Il est également fascinant d'aller se perdre dans la lecture des nombreux commentaires (passionnés, enragés, enthousiastes, scandalisés) auxquels des films comme Mothlight, Arnulf Rainer et Wavelength donnent lieu, où l'on retrouve une littérature haute en couleurs, des débats aussi animés que ceux que l'on pourrait retrouver dans une salle de classe après la présentation de ces films ${ }^{9}$. Nombre de commentaires d'ailleurs portent - je le mentionnais à propos d'Arnulf Rainer - sur le caractère tout à fait 
inapproprié du visionnement de ces films sur ces supports, et encouragent à tout prix que ces films soient vus dans de bonnes conditions, ou encore sont l'occasion de témoigner d'une expérience vécue, ailleurs et sur un bon support, de ces films. On trouve aussi, à côté des moqueries et des railleries vulgaires (qui sont les mêmes que l'on entend depuis 50 ans !), des tentatives d'éclaircissements pédagogiques, des francs et salutaires débats qui témoignent des dissensus sur le sens de l'art, des jeux d'esprit, des parodies, etc. qui sont emblématiques d'un rapport qu'on pourrait dire décomplexé avec la culture (haute ou basse), et autorise les appropriations, les plus diverses et variées.

\section{Mash-up, parodie, réappropriation}

15 Un des aspects les plus fascinants dans cette circulation du cinéma expérimental sur ces sites, est la culture du réemploi, du mash-up, du détournement souvent hilarant, iconoclaste ou idiot, de ces œuvres phares, réduites au rang de clip comme un autre. Hommage, parodie ou simple « repurposing ", YouTube permet de dresser un diagnostic $\mathrm{du}$ statut qu'ont acquis ces films. À chaque fois, ce sont des œuvres pionnières, décisives d'une certaine modernité du cinéma, équivalents pour les arts visuels de la Fontaine de Duchamp, ou, pour la musique, du 4'33" de John Cage. Ces remakes se greffent donc bel et bien, en même temps, à l'esprit postmoderne de l'échantillonnage, de l'appropriation, du remontage et du recyclage, déshistoricisé et décomplexé des "grandes œuvres".

16 On trouve, par exemple, de nombreuses versions avec accompagnement musical de Mothlight. Parfois, afin que la durée de la pièce corresponde à la durée du film, les «auteurs» sont allés jusqu'à ralentir le film ou le remonter (c'est notamment le cas d'un mash-up avec la pièce To Monk and Canatella de Portishead). Ces ajouts musicaux viennent semble-t-il pallier à ce qui apparaît souvent (pour des individus peu habitués au visionnement du cinéma muet, en silence) comme un manque dans les films de Brakhage, le son, le bruit, la musique. Ces versions permettent de faire se rencontrer un film et une musique préférée, de rendre hommage au film en se l'appropriant, etc. Le plaisir du monteur ou le trait d'esprit domine ici. Il y a également une version tout à fait étonnante d'Arnulf Rainer, affublée d'un extrait des Contes d'Hoffman d'Offenbach (il s'agit du repiquage de la même version sur YouTube dont il était question plus haut, et qui montre bien la circulation en circuit fermé des fichiers, ainsi que les effets de communauté particuliers qu'il induit). Pour antinomique qu'il soit (rien de plus éloigné $\mathrm{du}$ bombardement visuel et sonore d'Arnulf Rainer que la musique doucereuse d'Offenbach), ce montage télescope deux versions d'une culture perçue comme élitiste (cinéma d'avant-garde, musique classique).

On trouve également de nombreuses versions de Wavelength, parodies souvent sympathiques, qui font de l'esprit au carré avec l'esprit de Snow lui-même (que des générations d'étudiants de cinéma ont maudit pour la durée de son film). En réponse ironique à cette impatience des spectateurs, on se rappelle que le célèbre artiste canadien avait réalisé en 2003, en numérique, et avait rendu disponible en DVD, une version de 15 minutes de Wavelength, en découpant le film en trois sections et en superposant ces trois parties. Snow avait renommé le film: WVLNT or Wavelength for those who don't have the time. On retrouve cette version sur YouTube. Mais il existe depuis, au demeurant, des versions remontées ou accélérées de Wavelength (ou des 
hommages, des remontages), avec des durées très variables: de 4 minutes 50 , de 2 minutes 30, et même de 6 secondes (le très sympathique hommage à Wavelength, Short Wave (after Snow), réalisé par Brad Tinmouth, et mis en ligne en 2009). À ce titre, on pourrait faire le même exercice pour les très nombreuses et curieuses versions $d u$ Empire de Warhol (dont une version de 5 secondes), un autre film (invisible) connu pour sa durée excessive. Tous ces raccourcissements sont symptomatiques de la temporalité propre de YouTube, une " temporalité de la gratification instantanée ", une expression proposée par Lucas Hildebrand (2007b 49), et qui me semble en tout cas fondamentale pour comprendre le phénomène (car bien qu'il existe une version "intégrale » de Empire, tout comme du 45 minutes de Wavelength sur YouTube, qui en est véritablement le spectateur attentif?).

\section{Conclusion}

Toutes ces œuvres sont révélatrices des modalités de l'accès, de la réception et de la remédiation de ces images aujourd'hui. Cette circulation, déshistoricisée, ludique, idiote ou inventive du cinéma expérimental, que je me suis plu à traverser en accéléré dans cet article (en laissant beaucoup d'autres exemples de côté) doit il me semble être lue à l'aune d'un fait indéniable : nous traversons une époque où il devient, malgré les efforts remarquables de distributeurs comme Light Cone ou SixPack, des Cinémathèques, des salles d'art et d'essais et des collectifs de cinéma, de plus en plus difficile de voir ces films essentiels (et que dire de tous les autres) dans des bonnes conditions. Fuji ne produit plus de pellicule et Kodak annonce l'arrêt de la pellicule pour dans deux ans, nombre de laboratoires de développement ferment, on ne trouve plus de projecteurs $16 \mathrm{~mm}$ et $35 \mathrm{~mm}$ dans les salles de cinéma et souvent encore moins dans les salles de cours. Et malgré l'intérêt grandissant de la jeune génération pour les technologies analogiques, le numérique risque de mettre à mal la possibilité, d'ici quelques années, d'encore visionner ces films en pellicule. Il est sans doute légitime de se demander si, d'ici 15 ou 20 ans, la seule façon d'accéder à l'expérience (et non au film) de Mothlight ne serait par le biais de captations sauvages (comme on en retrouve par centaines de milliers, pour les concerts par exemple), réalisées à l'aide d'une petite caméra ou d'un téléphone. Ce n'est donc plus le film, mais l'expérience du film que l'on partage. C'est le cas de ce document remarquable, téléchargé sur YouTube par une spectatrice de la projection d'une très belle copie de Mothlight, en 2010, au Centre Pompidou : «I filmed this BRILLIANT FILM during its projection at Centre Pompidou, Paris on the 29th of sept 2010 ... There was a Conference about Nam June Paik, conference entitled AUTOUR de ZEN FOR FILM.» En plus de fournir le contexte particulier de la rencontre avec le film (dans un musée, autour d'une conférence sur Nam June Paik), la vidéo témoigne d'une captation enthousiaste, passionnée et émue, bien que résolument pixélisée, mal cadrée et, pour l'essentiel du film, hors foyer. Still. I'm glad it's there, somewhat. 


\section{BIBLIOGRAPHIE}

Bolter, Jay David, \& Grusin, Richard. Remediation: Understanding New Media. Cambridge: MIT Press, 2000.

Casetti, Francesco. «Cinema Lost and Found : Trajectories of Relocation. » Screening the past, 32.

24 octobre 2013. <http://www.screeningthepast.com/2011/11/cinema-lost-and-found-

trajectories-of-relocation>

Hidelbrand, Lucas. « Youtube: Where Cultural Memory and Copyright Converge. » Film Quarterly 61: 1 (automne 2007) 48-57.

Hildebrand, Lucas. "Grainy Days and Mondays : Superstar and Bootleg Aesthetics" Camera Obscura 19: 3 (2004) 56-91.

Perrot, Hugues \& Poli, Vincent. « Nouvelle cinéphilie (encore, encore). » Cahiers du cinéma, 703 (septembre 2014) 60-63.

Seyerl, Hito. « In Defense of The Poor Image. » e-flux 10 (novembre 2011). 24 octobre 2013.

<http://www.e-flux.com/journal/in-defense-of-the-poor-image>

FILMS

Arnulf Rainer, dir. Peter Kubelka, 1959.

Blowjob, dir. Andy Warhol, 1964.

Empire, dir. Andy Warhol, 1963.

Factory Archives, dir. Slater Bradley, 2001-2002.

La Région centrale, dir. Michael Snow, 1971.

Le retour à la raison, dir. Man Ray, 1923.

Mothlight, dir. Stan Brakhage, 1963.

Papillon, dir. Olivier Fouchard, 2006.

Superstar, dir. Todd Haynes, Iced Tea Productions, 1988.

The Flicker, dir. Tony Conrad, 1966.

The Garden of Earthly Delights, dir. Stan Brakhage, Canyon Cinema, 1981.

Wavelength, dir. Michael Snow, 1967.

Wavelength, Short Wave (after Snow), dir. Brad Tinmouth, 2009.

\section{NOTES}

1. On peut par exemple consulter cette version réduite à $1 \mathrm{~min} 03$ (au lieu de $3 \mathrm{~min}$ ), mise en ligne le 14 septembre 2007 par «Sara Bonaventura » et qui a été visionnée plus de 200000 fois : http://www.youtube.com/watch?v=XaGh0D2NXCA (page consultée le 23 octobre 2013)

2. J'emploie à dessein le terme "remédiation" (même si je l'utilise de façon plus élargie que Bolter et Grusin (2000) qui l'ont théorisé) plutôt que le plus populaire « relocation », consacré par Francesco Casetti (2011), puisque je crois que le changement de médium est plus déterminant que le déplacement de lieu, de format. 
3. On trouvera l'adresse ici: http://www.youtube.com/watch?v=iw1DVtFAz64 (consulté le 24 octobre 2013).

4. Parmi d'autres, ceux-ci : «Would you buy a used car from this man ?»; «It's clearly very influenced by the great masters of cinema: Alfred Hitchcock and John Ford "; " My favourite moment at 5:17»; It's like a baby trying to show a film - or, "YouTube" is totally malfunctioning...or, am I drunk ? But I am not... Somebody, an "artiste" is trying to sell us all down the river as bloody idiots. I for one, am not buying this shit!! » <http://www.youtube.com/ watch?v=iw1DVtFAz64> (consultée le 24 octobre 2013)

5. On le trouve à cette adresse-ci : http://www.youtube.com/watch?v=lzPwuP6AmCk (consulté le 24 octobre 2013).

6. Le cinéma expérimental est un bon lieu pour décrire et analyser les remédiations de l'image contemporaine : de la pellicule au VHS au DVD à Internet (pour faire court). Si, d'un côté, il s'agit d'élever au rang de figure esthétique les brouillages, les distorsions voire la ruine propre à chacun de ces médiums (la pellicule ne se dégrade pas de la même manière qu'une VHS, ni comme un DVD), ce sont aussi des manières d'offrir une description juste, phénoménologiquement parlant, de l'expérience de visionnement du cinéma expérimental. On peut penser à des films comme RIVER 0 (2002) de Jean-Claude Bustros, un found footage utilisant One Eyed Jack, seul film réalisé par Marlon Brando, et qui exploite les propriétés spécifiques au brouillage du signal numérique.

7. L'auteur du téléchargement (abusepotenti) prend d'ailleurs la peine de préciser : «Sorry for the hiss, second generation VHS dub. To some extent video degradation is intentional. » (http:// www.youtube.com/watch?v=-htjMLpmy9A).

8. Il n'a pas été possible d'analyser en détails les modalités spécifiques de ce site plus spécialisé et sa distinction avec YouTube. Je me permets toutefois de renvoyer le lecteur au papier de Hugues Perrot et Vincent Poli, « Nouvelle cinéphilie (encore, encore) », où il en est entre autres question (Perot \& Poli 2014, 60-63)

9. Parmi les commentaires (192, en date du 24 octobre 2013) les plus troublants, à propos de Wavelength on peut lire : "If this film was a race, I'd support its genocide.» (KKSAR, 11 votes pour) ; à propos de Mothlight, on peut lire : «The only way I can see Mothlight any more is on YouTube. That's a sign of the times. »

\section{RÉSUMÉS}

Pris au sens le plus large, le cinéma d'avant-garde ou expérimental, depuis Le retour à la raison de Man Ray jusqu'aux expérimentations contemporaines de Rose Lowder ou de Peter Tscherkassky, en passant par Snow, Sharits ou Brakhage, toujours cherché à explorer les spécificités du médium cinématographique : chimie de la lumière sur la pellicule, nature photogrammatique de l'image en mouvement, expérience de la durée, de la projection, matérialité du film, etc. Tous ces éléments, toutes ces conditions sont nécessaires afin que le film - et ceci, davantage que pour un film documentaire ou de fiction narratif - libère si l'on veut son plein potentiel. L'expérience du film est inscrite dans sa matérialité, dans le dispositif cinématographique de base. Cela explique entre autres que, malgré le travail de dissémination important de distributeurs DVD comme Re:Voir, Criterion (les coffrets Brakhage, Frampton), Mystic Fire, Index ou LuxFilm, des pans entiers du cinéma expérimental ne sont "officiellement » encore visibles qu'en pellicule $16 \mathrm{~mm}$ ou $35 \mathrm{~mm}$. La rareté de ces films aura, en retour, engendré une grande plus-value auprès des 
collectionneurs et des amateurs de films sur le Web. Aussi, de YouTube à MUBI ou Ubuweb, en passant par des sites plus confidentiels comme Karagarga, on retrouve un nombre impressionnant de films expérimentaux. Ces films subissent des remédiations numériques défigurantes, des réappropriations sauvages et des remix tout aussi fascinants qu'inquiétants, qui témoignent bien d'un état contemporain de la circulation des images. Il s'agira, dans le cadre de cet article, de réfléchir aux modalités de cette circulation à partir d'un ensemble d'exemples pertinents.

Taken at large, experimental or avant-garde cinema has always tried to explore the specificities of the cinematic medium: chemistry of light, photogrammatic nature of moving images, duration, projection, film materiality, etc. All these elements are required for the film to liberate its own potential. This explains, notwithstanding the efforts and value of the dissemination by DVD distributors such as Criterion, Re:Voir, Index, LuxFilm, that many of these films are still "officially" only available on celluloid (16 $\mathrm{mm}$ or $35 \mathrm{~mm}$ ). This scarcity has produced, in return, an incredible value for these films among collectors and film fans on the Web. From YouTube to MUBI or Ubuweb, we find an impressive array of experimental films that were never made to be seen in this way. These films endure violent digital remediation, savage reappropriations and remixes that are both fascinating and worrisome, and that all testify to a contemporary state of the circulation of images. In this article, we wish to reflect upon these modalities of cinematic circulation using a series of relevant examples.

\section{INDEX}

Mots-clés : cinéma expérimental, YouTube, cinéma numérique, remédiation, circulation des images, esthétique bootleg

Keywords : experimental cinema, YouTube, Digital Cinema, remediation, circulation of images, nostalgia, bootleg aesthetic

\section{AUTEURS}

\section{ANDRÉ HABIB}

Université de Montréal

Professeur agrégé

andre.habib@umontreal.ca 